\title{
Analysis of viral load in different specimen types and serum antibody levels of COVID-19 patients
}

Ling $\mathrm{Li}^{1,2 \dagger}{ }^{+}$, Chianru Tan ${ }^{3 \dagger}$, Jia Zeng ${ }^{4,5+}$, Chen Luo ${ }^{1,2}$, Shi Hu${ }^{6}$, Yanke Peng ${ }^{3}$, Wenjuan Li ${ }^{1,7}$, Zhixiong Xie ${ }^{5,8}$, Yueming Ling ${ }^{5,9}$, Xuejun Zhang ${ }^{1}$, E. Deng ${ }^{1}$, Haixia Xu ${ }^{1,2}$, Jue Wang ${ }^{1,2}$, Yudi Xie ${ }^{1,2}$, Yaling Zhou ${ }^{1,2}$, Wei Zhang ${ }^{5,10^{*}}$, Yong $\mathrm{GuO}^{3^{*}}$ and Zhong Liu ${ }^{1,2^{*}}$

\begin{abstract}
Background: COVID-19 has caused a global pandemic and the death toll is increasing. However, there is no definitive information regarding the type of clinical specimens that is the best for SARS-CoV-2 detection, the antibody levels in patients with different duration of disease, and the relationship between antibody level and viral load.

Methods: Nasopharyngeal swabs, anal swabs, saliva, blood, and urine specimens were collected from patients with a course of disease ranging from 7 to 69 days. Viral load in different specimen types was measured using droplet digital PCR (ddPCR). Meanwhile, anti-nucleocapsid protein (anti-N) IgM and IgG antibodies and anti-spike protein receptorbinding domain (anti-S-RBD) IgG antibody in all serum samples were tested using ELISA.

Results: The positive detection rate in nasopharyngeal swab was the highest (54.05\%), followed by anal swab (24.32\%), and the positive detection rate in saliva, blood, and urine was $16.22 \%, 10.81 \%$, and $5.41 \%$, respectively. However, some patients with negative nasopharyngeal swabs had other specimens tested positive. There was no significant correlation between antibody level and days after symptoms onset or viral load.

Conclusions: Other specimens could be positive in patients with negative nasopharyngeal swabs, suggesting that for patients in the recovery period, specimens other than nasopharyngeal swabs should also be tested to avoid false negative results, and anal swabs are recommended. The antibody level had no correlation with days after symptoms onset or the viral load of nasopharyngeal swabs, suggesting that the antibody level may also be affected by other factors.
\end{abstract}

Keywords: COVID-19, Viral load, Droplet digital PCR, Nasopharyngeal swab

\footnotetext{
*Correspondence: zhangweismmu@126.com; yongguo@tsinghua.edu.cn; liuz@ibt.pumc.edu.cn

${ }^{\dagger}$ Ling Li, Chianru Tan and Jia Zeng contributed equally to this work ${ }^{1}$ Institute of Blood Transfusion, Chinese Academy of Medical Sciences and Peking Union Medical College, Chengdu 610052, Sichuan, People's Republic of China

${ }^{3}$ Department of Biomedical Engineering, School of Medicine, Tsinghua University, 30 Shuangqing Road, Beijing 100084, People's Republic of China

${ }^{10}$ Department of Respiratory and Critical Care Medicine, First Affiliated Hospital, Second Military Medical University, 168\# Changhai Rd, Shanghai 200433, People's Republic of China

Full list of author information is available at the end of the article
}

\section{Background}

The recent emergence of the severe acute respiratory syndrome coronavirus 2 (SARS-CoV-2) poses a serious threat to human health. SARS-CoV-2 can cause asymptomatic infections, mild self-limiting respiratory diseases, and severe progressive pneumonia (resulting in shock, acute respiratory distress syndrome (ARDS), acute heart injury, acute kidney injury, and death) $[1,2]$. World Health Organization (WHO) named the disease caused by SARS-CoV-2 infection the coronavirus disease 2019 (COVID-19), which has rapidly expanded across the

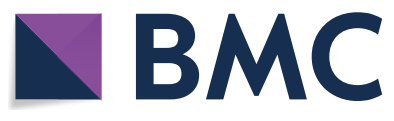

(c) The Author(s) 2021. This article is licensed under a Creative Commons Attribution 4.0 International License, which permits use, sharing, adaptation, distribution and reproduction in any medium or format, as long as you give appropriate credit to the original author(s) and the source, provide a link to the Creative Commons licence, and indicate if changes were made. The images or other third party material in this article are included in the article's Creative Commons licence, unless indicated otherwise in a credit line to the material. If material is not included in the article's Creative Commons licence and your intended use is not permitted by statutory regulation or exceeds the permitted use, you will need to obtain permission directly from the copyright holder. To view a copy of this licence, visit http://creativeco mmons.org/licenses/by/4.0/. The Creative Commons Public Domain Dedication waiver (http://creativecommons.org/publicdomain/ zero/1.0/) applies to the data made available in this article, unless otherwise stated in a credit line to the data. 
globe. A rapid and reliable diagnosis of COVID-19 is critical for control of this pandemic.

Quantitative reverse transcription polymerase chain reaction (RT-qPCR) is the main diagnostic method to identify patients with COVID-19. Proper specimen collection is important for the diagnosis [3]. Studies have found that SARS-CoV-2 nucleic acid could be detected in nasopharyngeal swabs, sputum, saliva, blood, urine, and anal swabs/feces of COVID-19 patients [4], and the positive detection rate of sputum was the highest, followed by nasopharyngeal swabs [5]. However, not all patients are able to produce sputum, especially elderly patients and patients with endotracheal intubation, which makes it difficult to extract sputum. In addition, the high viscosity of sputum makes it difficult to extract nucleic acids. Therefore, most specimens collected at present are nasopharyngeal swabs. However, poor quality of nasopharyngeal swabs collection could contribute to false-negative results, and it has been reported that the rectal/anal swabs from some patients who in the recovery stage were persistently tested positive after the nasopharyngeal testing was negative $[6,7]$. Thus, further research is needed to establish which specimen types are most suitable for SARS-CoV-2 nucleic acid detection.

In addition to nucleic acid detection, virus-specific antibody detection is of great significance for auxiliary diagnosis, differential diagnosis, and monitoring the disease progression and treatment effect. Researchers have studied antibody kinetics to determine the seroconversion rate and median seroconversion time of patients with COVID-19 [8] as well as the relationship between disease severity and the antibody level [9]. However, the antibody levels in patients with different duration of disease and the relationship between antibody level and viral load remained unclear.

In this study, we collected nasopharyngeal swabs, anal swabs, saliva, blood, and urine specimens of COVID-19 patients with a disease course of 7-69 days, and used droplet digital (ddPCR) to detect SARS-CoV-2 in these different specimens. We also measured the anti-nucleocapsid protein (anti-N) IgM and IgG levels in the serum of these patients and the titer of anti-spike protein receptor-binding domain (anti-S-RBD) IgG to study the correlation between antibody production and viral load in COVID-19 patients.

\section{Methods}

\section{Ethics statement}

The study was approved by the Ethics Committee of the Institute of Blood Transfusion, Chinese Academy of Medical Sciences \& Peking Union Medical College. Written informed consent was obtained from each study participant.

\section{Participants}

A total of 185 samples from 37 patients with COVID-19 were collected in Maternal and Child Health Care Hospital of Hubei Province (Guanggu District) between March 17 and March 24, 2020. Nasopharyngeal swab, anal swab, saliva, blood, and urine were collected from each patient. Written informed consent was obtained from all patients before the study.

\section{Clinical classification of COVID-19}

The diagnosis was based on the Diagnosis and Treatment Protocol for COVID-19 (trial version 7) established by the National Health Commission of the People's Republic of China [10]. The clinical classification of COVID-19 was as follows: (1) Mild: mild clinical symptoms with no sign of pneumonia on imaging. (2) Moderate: showing fever and respiratory symptoms with radiological findings of pneumonia. (3) Severe: adult cases meeting any of the following criteria: (a) respiratory distress ( $\geq 30$ breaths/ min); (b) oxygen saturation $\leq 93 \%$ at rest; (c) arterial partial pressure of oxygen $\left(\mathrm{PaO}_{2}\right)$ /fraction of inspired oxygen $\left(\mathrm{FiO}_{2}\right) \leq 300 \mathrm{mmHg}(1 \mathrm{mmHg}=0.133 \mathrm{kPa})$; (d) cases with chest imaging that shows obvious lesion progression within $24-48 \mathrm{~h}>50 \%$. (4) Critical: meeting any of the following criteria: (a) respiratory failure and mechanical ventilation is required; (b) shock; (c) with other organ failure that requires ICU care.

\section{Specimen collection and transportation}

Nasopharyngeal swab, anal swab, saliva, blood, and urine were collected and stored at $4{ }^{\circ} \mathrm{C}$ until use. Sampling methods were as follows:

1. Nasopharyngeal swab: The patient was instructed to rinse his/her mouth with water, and then a swab was inserted through the nostril parallel to the palate. The swab was left in the nasopharynx for $15 \mathrm{~s}$ and gently rotated three times. The swab was then withdrawn and placed into a collection tube.

2. Anal swab: A sampling swab was soaked in normal saline, and then inserted $2-3 \mathrm{~cm}$ deep into the anus. The swab was used to wipe the fold around the anus or gently rub against the anal opening. The swab was withdrawn and placed into a tube containing normal saline.

3. Saliva: In the morning, the patient was instructed to rinse his/her mouth with water and then rest for $10 \mathrm{~min}$. Then, the initial saliva was discarded, and the patient spit at least $2 \mathrm{ml}$ of saliva into the collection tube. If the patient was not able to produce a sufficient amount of saliva, oral and tongue exercise was 
used to promote saliva secretion. The cap was tightened after collection and the tube was turned upside down five times.

4. Blood: A vacuum negative-pressure blood collection tube was used to collect $5 \mathrm{ml}$ of blood each in a heparin anticoagulation tube and an EDTA anticoagulation tube. The blood specimens were left at room temperature for $30 \mathrm{~min}$.

5. Urine: Morning urine was collected. The initial urine was discarded and the middle urine was collected. To collect urine from a urine catheter, the urinary catheter was clamped and the sampling site was disinfected with alcohol. The catheter was punctured with a sterile syringe to draw urine into a collection tube.

\section{RNA extraction and ddPCR detection of viral load in the different specimens}

Samples were collected and soaked in $1000 \mu \mathrm{l}$ PBS buffer. Viral RNA was extracted within $2 \mathrm{~h}$ according to the manufacturer's instructions using the MagPure Viral Nucleic Acid Duo Kit (Magen, Guangzhou, China).

The 185 samples were tested using ddPCR. The ddPCR was performed using a SARS-CoV-2 nucleic acid detection kit (ddPCR) (TargetingOne, Beijing, China) and a TD-1 ${ }^{\text {TM }}$ Droplet Digital ${ }^{\mathrm{TM}}$ PCR system (TargetingOne, Beijing, China, licensed in China, registration number: 20170025, 20192220517) following the manufacturer's instructions.

\section{Detection of anti-N IgM and IgG antibodies}

Anti-N IgM and IgG antibodies in all serum samples except for one patient were tested by the enzyme-linked immunosorbent assay (ELISA) according to the manufacturer's instructions. The anti-N IgM and IgG antibody detection kits were purchased from domestic pharmaceutical group Inc (China).

\section{Titer of anti-S-RBD IgG antibody}

Anti-S-RBD IgG antibody in all serum samples except for one patient were tested using the ELISA assay as previously described [11]. Results were reported as the ratio of a sample's OD value (S) to the cut-off value (CO), i.e., the $\mathrm{S} / \mathrm{CO}$ value. Samples were diluted serially and tested by the ELISA assay. Titers were reported as the highest dilution when the ELISA assay was still positive, ranging between 1:160 and 1:2560 (ELISA endpoint dilution titers).

\section{Results}

Basic characteristics of the patients

The median age of the 37 patients was 57 years (range $30-94$ years), including 26 women and 11 men. Most of the patients had moderate cases, with four patients with severe or critical cases. Table 1 shows the baseline characteristics and routine blood results of the 37 patients with COVID-19.

\section{Positive detection rates and viral load of samples from different sites}

As shown in Fig. 1a, b, the nasopharyngeal swab had the highest nucleic acid positivity rate at $54.05 \%$ (20/37), followed by the anal swab at $24.32 \%(9 / 37)$, and the saliva, blood, and urine were at $16.22 \%(6 / 37)$, $10.81 \%(4 / 37)$, and $5.41 \%(2 / 37)$, respectively. As shown in Fig. 1a, two patients had positive anal swabs but negative nasopharyngeal swabs, and one patient had only a positive anal swab among all specimens. Three patients had positive saliva specimens but negative nasopharyngeal swabs, with one of them having no other positive specimens. One patient was tested positive only in the blood. Of the two patients with positive urine nucleic acid, one patient had no other positive specimens and the other was also positive for the saliva and urine specimens. As shown in Fig. 1b, the mean viral loads of nasopharyngeal swabs, anal swabs, saliva, blood, and urine specimens were $16,224 \pm 67,507$, $20 \pm 26,5677 \pm 13,647,16 \pm 9$, and $5.1 \pm 0$ copies/test, respectively.

\section{Relationship between antibody levels and days after symptoms onset}

Figure 2a demonstrates the relationship between anti$\mathrm{N}$ IgM/IgG antibody levels and days after symptoms onset. Most patients had low levels of IgM antibodies, with only 9 patients $(25 \%)$ having positive anti-N IgM $(\mathrm{S} / \mathrm{CO}$ ratio $\geq 1)$. All patients had positive anti-N IgG (100\%), but no significant correlation was observed between anti-N IgM/IgG levels and days after symptoms onset. Figure $2 \mathrm{~b}$ shows the relationship between anti-S-RBD IgG antibody level and days after symptoms onset; 7 patients (19\%) had an anti-S-RBD IgG titer of less than 1:640, and again, no significant correlation between anti-S-RBD IgG level and days after symptoms onset was observed.

\section{Relationship between antibody levels and viral load of nasopharyngeal swabs}

Figure 3a demonstrates the relationship between anti-N IgM/IgG and viral load of the nasopharyngeal swab. For specimens with anti-N IgM levels above the threshold 


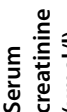

ฐ

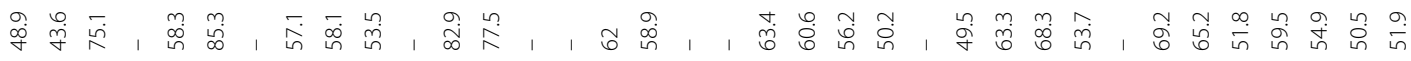

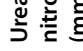

离 $\overline{\mathrm{\varepsilon}}$

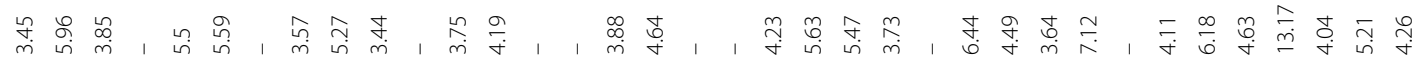

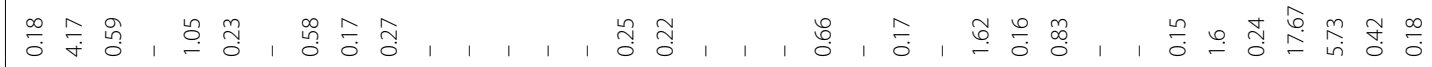

공 $\infty$

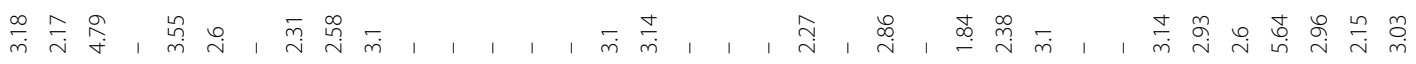

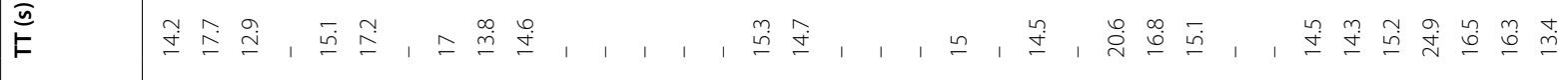

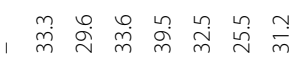
$\stackrel{+}{\rightleftarrows} \simeq, \stackrel{\varrho}{=} \stackrel{\varrho}{=}, \stackrel{m}{=} \simeq$ $\stackrel{-}{\circ}$

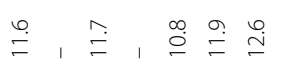
$\stackrel{n}{=} \stackrel{n}{=} \stackrel{m}{=} \stackrel{\stackrel{0}{=}}{=} \stackrel{n}{=}$ $\stackrel{\bar{\sigma}}{\stackrel{\bar{\sigma}}{\circ}}$ , 논 는 , ,

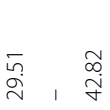
$\stackrel{2}{-}$ , 늘 ㅇํํ

, 1

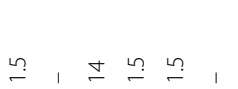

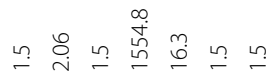

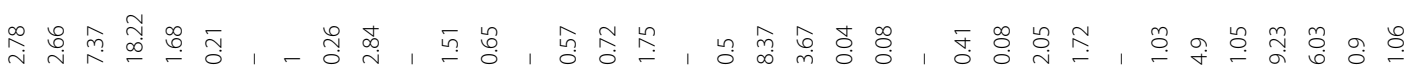
출

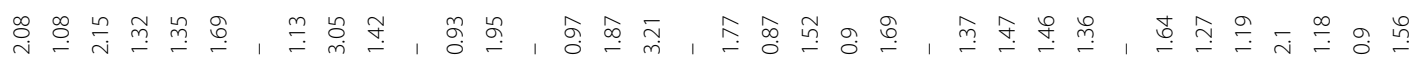
is

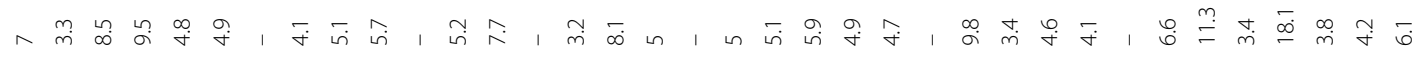

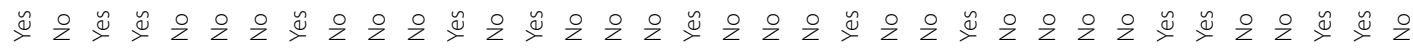

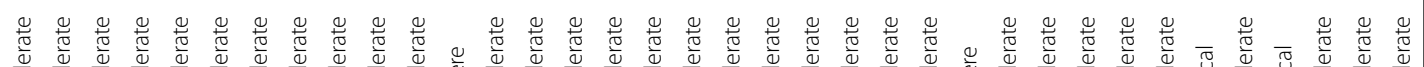

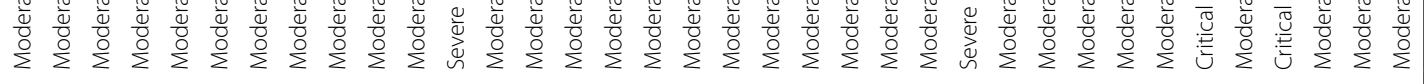

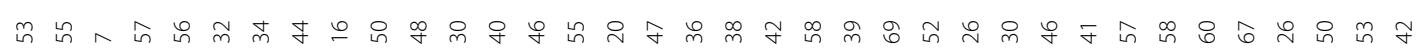

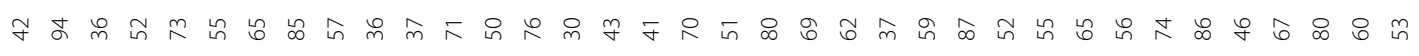
ш 㤩 
$(\mathrm{S} / \mathrm{CO}$ ratio $\geq 1)$, there is a tendency for the antibody level to decrease as viral load increased. Whereas the anti-N IgG level did not have a significant correlation with viral load, and the IgG level was extremely low in a patient with a viral load greater than $10^{5}$ copies/test. Figure 3b shows the anti-S-RBD IgG level in relation to nasopharyngeal swab viral load, and again, no significant correlation was observed between the anti-S-RBD IgG level and viral load.

\section{Discussion}

This study collected multiple types of specimens from COVID-19 patients with a disease course of 7-69 days, including nasopharyngeal swabs, anal swabs, saliva, blood, and urine specimens. ddPCR was used for nucleic acid detection and absolute quantification of these specimens. ELISA was used to detect the anti-N IgM/IgG and the anti-S-RBD IgG in the serum samples of these patients.

The results of nucleic acid testing of specimens showed that the positive detection rate of nasopharyngeal swabs was the highest, and the average viral load of nasopharyngeal swabs was also the highest. This finding is consistent with the results of existing reports [4]. Research has pointed out that viral infection may damage the gastrointestinal tract, so in the later stages of the disease, the virus may be detected in anal swabs [12], and anal swabs/ fecal specimens may remain positive after the nasopharyngeal swabs become negative $[13,14]$. The present study also observed this phenomenon, and the positive detection rate of anal swabs was relatively high, reaching $24 \%$. A previous study has shown that the positive detection rate of saliva specimens is high, up to $61.5 \%$ [15]. The study found that viral nucleic acids could be detected in posterior oropharyngeal saliva specimens, and a better positive percent agreement was observed in specimens obtained within 7 days after symptoms onset. However, the positive detection rate of saliva specimens in our study was only $16 \%$, which may be related to the sampling method and the day of specimen collection [16]. In summary, our results showed that the nasopharyngeal site is the best for the detection of SARS-CoV-2. Meanwhile, the results also suggested that nucleic acid testing for convalescent patients should be done in nasopharyngeal swabs plus other specimens to get a more accurate diagnosis of full recovery from coronavirus infection since other specimens from patients with negative nasopharyngeal test result were tested positive in this study. It is recommended to test anal swab, because in this study, except for nasopharyngeal swabs, SARS-CoV-2 was mostly detected in anal swabs. Moreover, several studies have characterized the presence of live virus in feces 


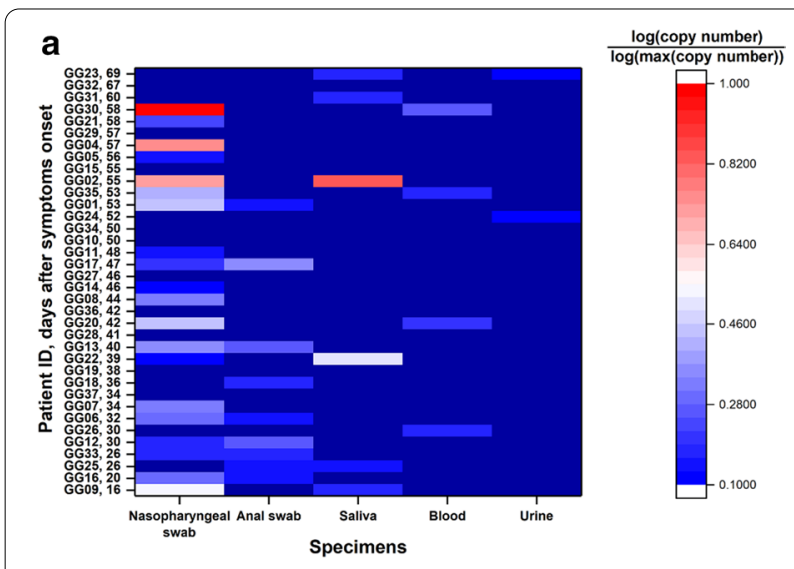

b

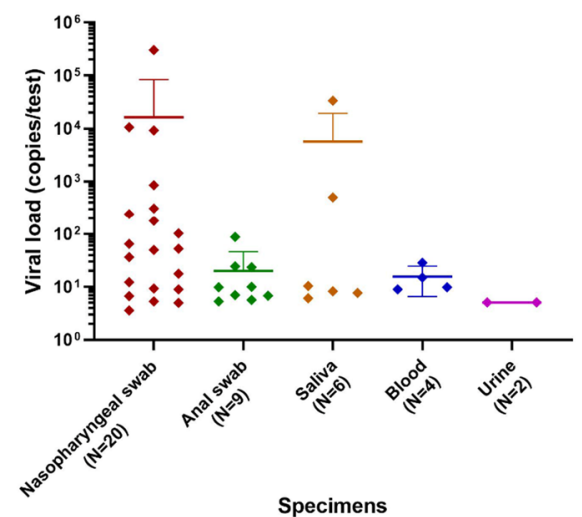

Fig. 1 Viral nucleic acid test results. a Nucleic acid test results of different specimens from different patients. The $x$-axis is the specimen, and the $y$-axis is the patients and days after symptoms onset. The $y$-axis shows the number of days of illness in descending order from top to bottom. Each cell is colored by viral load. b Viral load of different specimens from different patients. The mean copy number and standard deviation are shown in the figure
[17-19], indicating that SARS-CoV-2 may be transmitted by fecal route. Notably, Wang et al. [19] failed to isolate virus in feces specimens collected at later time points of disease onset. Therefore, the infectivity of SARS-CoV-2 in different specimens at different time points needs further investigation to better guide the clinical management of COVID-19 patients.

The test results of the antibody level and titer showed that there was no correlation between anti-N IgM/IgG or anti-S-RBD IgG levels with days after symptoms onset or the viral load of nasopharyngeal swabs, which suggested that the antibody level may also be influenced by other factors. The existing literature pointed out that the median (interquartile range, IQR) seroconversion time of anti-N IgM and IgG is 10 days (7-14 days) while the median (IQR) seroconversion time of anti-S-RBD IgG is 13 days (9-17 days). The duration of IgM is short as the IgM level attenuates after reaching the peak at 14 days (9-23 days) while the duration of IgG is longer than IgM [20]. This could explain the results of the present study: most of the patients' course of disease was more than 2 weeks, so the level of anti-N IgM was low, while anti-N IgG and anti-S-RBD IgG were at a high level.

This study has some limitations. First, the sample size of this study is small, which may cause some correlations fail to be detected. Second, most of the patients in this study had moderate cases, with only four severe or critical patients, so no further multi-parameter analyses could be conducted. Third, the different specimens from each patient were only sampled once and tested once, which may have caused false results due to some pre-analytical and analytical errors.
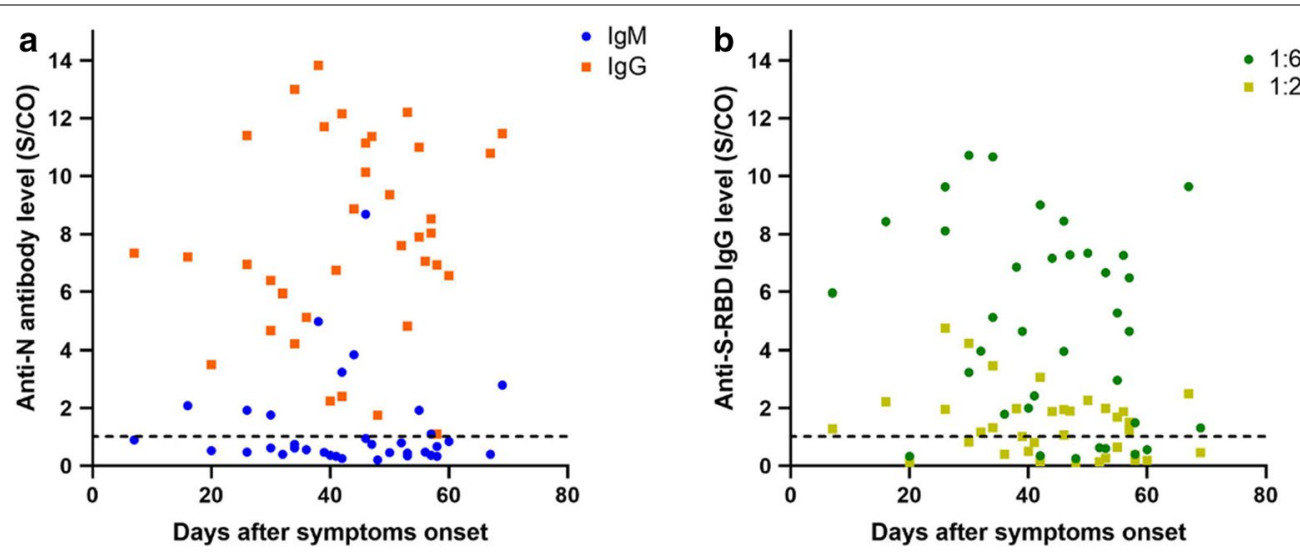

Fig. 2 Relationship between $\mathbf{a}$ anti-N IgG/lgM antibody levels or $\mathbf{b}$ anti-S-RBD IgG level and days after symptoms onset. The dashed line shows the threshold (S/CO ratio $\geq 1)$ 

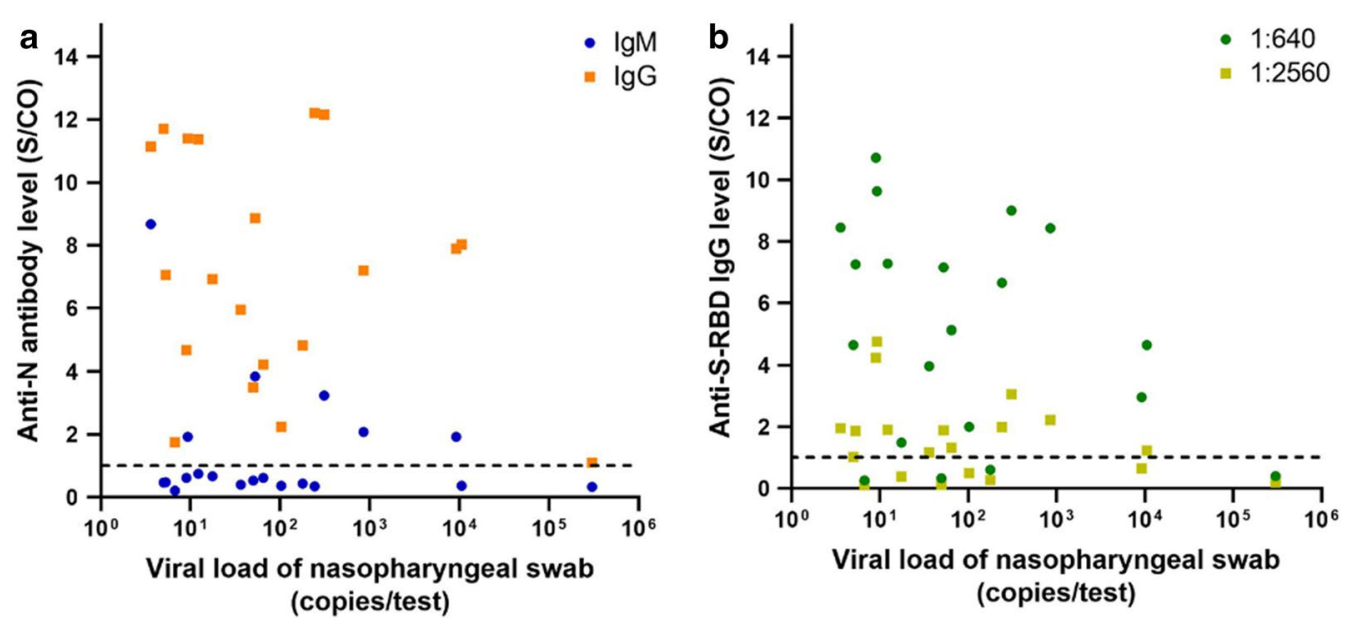

Fig. 3 Relationship between a anti-N IgG/lgM antibody levels or $\mathbf{b}$ anti-S-RBD Ig level and viral load of the nasopharyngeal swab. The dashed line shows the threshold (S/CO ratio $\geq 1$ )

\section{Conclusion}

COVID-19 is a new infectious disease, and a better understanding of it can help us better prevent and treat the disease. This study analyzed the nucleic acid positive detection rate and viral load of different types of specimens from COVID-19 patients and studied the relationship of antibody levels with days after symptoms onset and viral load. The findings of this study provided the scientific basis for understanding the antibody responses and improving viral nucleic acid sampling and detection.

\begin{abstract}
Abbreviations
SARS-CoV-2: Severe acute respiratory syndrome coronavirus 2; ARDS: Acute respiratory distress syndrome; WHO: World Health Organization; COVID19: Coronavirus disease 2019; RT-qPCR: Quantitative reverse transcription polymerase chain reaction; ddPCR: Droplet digital polymerase chain reaction; anti-N: Anti-nucleocapsid protein; anti-S-RBD: Anti-spike protein receptorbinding domain; ELISA: Enzyme-linked immunosorbent assay; S: Sample's OD value; CO: Cut-off value.
\end{abstract}

\section{Acknowledgements}

Not applicable.

\section{Authors' contributions \\ $L L$ and $J Z$ designed the study and collected samples; $L L$ and $C T$ are the major contributors in writing the manuscript; $L L, C T, J Z C L, S H, Y P, W L, Z X, Y L, X Z, E D$, HX, JW, YX and YZ collected the clinical data and analyzed data. WZ, YG, ZL conceived the idea for the study and reviewed manuscript. All authors read and approved the final manuscript.}

\section{Funding}

This work was supported by the CAMS Innovation Fund for Medical Sciences (CIFMS) (Grant Nos. 2016-12M-3-024 and 2020-12M-CoV19-006), Tsinghua University Initiative Scientific Research Program, Beijing Science and Technology Project (Grant No. Z201100005420025) and Tsinghua University Spring Breeze Fund (Grant No. 2020Z99CFG010).

Availability of data and materials

All data generated or analyzed during this study are included in this article.

\section{Ethics approval and consent to participate}

The study was approved by the Ethics Committee of the Institute of Blood Transfusion, Chinese Academy of Medical Sciences \& Peking Union Medical College. Written informed consent was obtained from each study participant.

\section{Consent for publication}

Not applicable.

\section{Competing interests}

The authors declare that they have no competing interests.

\section{Author details}

${ }^{1}$ Institute of Blood Transfusion, Chinese Academy of Medical Sciences and Peking Union Medical College, Chengdu 610052, Sichuan, People's Republic of China. ${ }^{2}$ Key Laboratory of Transfusion Adverse Reactions, Chinese Academy of Medical Sciences, Chengdu 610052, Sichuan, People's Republic of China. ${ }^{3}$ Department of Biomedical Engineering, School of Medicine, Tsinghua University, 30 Shuangqing Road, Beijing 100084, People's Republic of China. ${ }^{4}$ Department of Aviation Disease, Naval Medical Center of PLA, Second Military Medical University, Shanghai 200052, People's Republic of China. ${ }^{5}$ The Maternal and Child Health Hospital of Hubei Province, Guanggu District, Wuhan 430070, Hubei, People's Republic of China. ${ }^{6}$ Department of Biophysics, College of Basic Medical Sciences, Second Military Medical University, Shanghai 200433, People's Republic of China. ${ }^{7}$ Anhui Medical University, Hefei 230032, People's Republic of China. ${ }^{8}$ Department of Clinical Laboratory Science of NO. 909 Hospital of PLA Joint Support Force, Zhangzhou 363000, People's Republic of China. ${ }^{9}$ Department of Clinical Laboratory Science of NO. 910 Hospital of PLA Joint Support Force, Quanzhou 362000, People's Republic of China. ${ }^{10}$ Department of Respiratory and Critical Care Medicine, First Affiliated Hospital, Second Military Medical University, 168\# Changhai Rd, Shanghai 200433, People's Republic of China.

Received: 17 October 2020 Accepted: 29 December 2020

Published online: 07 January 2021

\section{References}

1. Huang C, Wang Y, Li X, Ren L, Zhao J, Hu Y, Zhang L, Fan G, Xu J, Gu X, et al. Clinical features of patients infected with 2019 novel coronavirus in Wuhan China. Lancet. 2020;395(10223):497-506.

2. Wang D, Hu B, Hu C, Zhu F, Liu X, Zhang J, Wang B, Xiang H, Cheng Z, Xiong Y, et al. Clinical characteristics of 138 hospitalized patients with 
2019 novel coronavirus-infected pneumonia in Wuhan China. JAMA. 2020;323:1061-9.

3. Jin YH, Cai L, Cheng ZS, Cheng H, Deng T, Fan YP, Fang C, Huang D, Huang $L Q$, Huang $Q$, et al. A rapid advice guideline for the diagnosis and treatment of 2019 novel coronavirus (2019-nCoV) infected pneumonia (standard version). Mil Med Res. 2020;7(1):4.

4. Boger B, Fachi MM, Vilhena RO, Cobre AF, Tonin FS, Pontarolo R. Systematic review with meta-analysis of the accuracy of diagnostic tests for COVID-19. Am J Infect Control. 2020:49:21-9.

5. Yu F, Yan L, Wang N, Yang S, Wang L, Tang Y, Gao G, Wang S, Ma C, Xie $R$, et al. Quantitative detection and viral load analysis of SARS-CoV-2 in infected patients. Clin Infect Dis. 2020;71:793-8.

6. Xu Y, Li X, Zhu B, Liang H, Fang C, Gong Y, Guo Q, Sun X, Zhao D, Shen $J$, et al. Characteristics of pediatric SARS-CoV-2 infection and potential evidence for persistent fecal viral shedding. Nat Med. 2020;26(4):502-5.

7. Zhang B, Liu S, Dong Y, Zhang L, Zhong Q, Zou Y, Zhang S. Positive rectal swabs in young patients recovered from coronavirus disease 2019 (COVID-19). J Infect. 2020;81:e49-52.

8. Zhao J, Yuan Q, Wang H, Liu W, Liao X, Su Y, Wang X, Yuan J, Li T, Li J, et al. Antibody responses to SARS-CoV- 2 in patients of novel coronavirus disease 2019. Clin Infect Dis. 2020;71:2027-34.

9. Wang Y, Zhang L, Sang L, Ye F, Ruan S, Zhong B, Song T, Alshukairi AN, Chen $R$, Zhang Z, et al. Kinetics of viral load and antibody response in relation to COVID-19 severity. J Clin Invest. 2020;130:5235-44.

10. National Health Commission. Diagnosis and treatment protocol for novel coronavirus pneumonia (Trial Version 7). Chin Med J. 2020;133(9):1087-95.

11. Li L, Yang R, Wang J, Lv Q, Ren M, Zhao L, Chen H, Xu H, Xie S, Xie J, et al. Feasibility of a pilot program for COVID-19 convalescent plasma collection in Wuhan China. Transfusion. 2020;60(8):1773-7.

12. Tian $Y$, Rong L, Nian W, He Y. Review article: gastrointestinal features in COVID-19 and the possibility of faecal transmission. Aliment Pharmacol Ther. 2020;51(9):843-51.
13. Kipkorir V, Cheruiyot I, Ngure B, Misiani M, Munguti J. Prolonged SARSCov-2 RNA detection in anal/rectal swabs and stool specimens in COVID19 patients after negative conversion in nasopharyngeal RT-PCR test. J Med Virol. 2020;92:2328-31.

14. Fan Q, Pan Y, Wu Q, Liu S, Song X, Xie Z, Liu Y, Zhao L, Wang Z, Zhang Y, et al. Anal swab findings in an infant with COVID-19. Pediatr Investig. 2020;4(1):48-50.

15. Cheuk S, Wong Y, Tse H, Siu HK, Kwong TS, Chu MY, Yau FYS, Cheung IYY, Tse CWS, Poon KC, et al. Posterior oropharyngeal saliva for the detection of SARS-CoV-2. Clin Infect Dis. 2020. https://doi.org/10.1093/cid/ciaa797.

16. Baghizadeh Fini M. Oral saliva and COVID-19. Oral Oncol. 2020;108:104821.

17. Zhang Y, Chen C, Song Y, Zhu S, Wang D, Zhang H, Han G, Weng Y, Xu J, Xu J, et al. Excretion of SARS-CoV-2 through faecal specimens. Emerg Microbes Infect. 2020;9(1):2501-8.

18. Xiao F, Sun J, Xu Y, Li F, Huang X, Li H, Zhao J, Huang J, Zhao J. Infectious SARS-CoV-2 in feces of patient with severe COVID-19. Emerg Infect Dis J. 2020;26(8):1920.

19. Wang W, Xu Y, Gao R, Lu R, Han K, Wu G, Tan W. Detection of SARS-CoV-2 in different types of clinical specimens. JAMA. 2020;323(18):1843-4.

20. Huang J, Mao T, Li S, Wu L, Xu X, Li H, Xu C, Su F, Dai J, Shi J, et al. Long period dynamics of viral load and antibodies for SARS-CoV-2 infection: an observational cohort study. MedRxiv. 2020. https://doi. org/10.1101/2020.04.22.20071258.

\section{Publisher's Note}

Springer Nature remains neutral with regard to jurisdictional claims in published maps and institutional affiliations.
Ready to submit your research? Choose BMC and benefit from:

- fast, convenient online submission

- thorough peer review by experienced researchers in your field

- rapid publication on acceptance

- support for research data, including large and complex data types

- gold Open Access which fosters wider collaboration and increased citations

- maximum visibility for your research: over 100M website views per year

At BMC, research is always in progress.

Learn more biomedcentral.com/submissions 